\title{
Psychoneuroendocrinology and Clinical Psychology
}

\author{
Susanne Fischer ${ }^{\mathrm{a}}$, Ulrike Ehlert ${ }^{\mathrm{a}}$ \\ [a] Institute of Psychology, Clinical Psychology and Psychotherapy, University of Zurich, Zurich, Switzerland.
}

Clinical Psychology in Europe, 2019, Vol. 1(2), Article 33030, https://doi.org/10.32872/cpe.v1i2.33030

Received: 2019-01-11 • Accepted: 2019-03-01 • Published (VoR): 2019-06-28

Handling Editor: Winfried Rief, Philipps-University of Marburg, Marburg, Germany

Corresponding Author: Susanne Fischer, University of Zurich, Institute of Psychology, Clinical Psychology and Psychotherapy, Binzmuehlestrasse 14 / Box 26, 8050 Zurich, Switzerland. E-mail: s.fischer@psychologie.uzh.ch

\begin{abstract}
Background: Hormones impact on cognition, emotions, and behaviour. Given that mental disorders are defined by abnormalities in these very same domains, clinical psychologists may benefit from learning more about alterations in endocrine systems, how they can contribute to symptoms commonly experienced by patients, and how such knowledge may be put to use in clinical practice.
\end{abstract}

Method: The aim of the present scientific update was to provide a brief overview of endocrine research relevant to the aetiology, diagnostics, and treatment of mental disorders, including some of the latest studies in this area.

Results: Hormones appear to be intrinsic to the development and maintenance of mental disorders. Oxytocin is involved in social cognition and behaviour and as such may be relevant to mental disorders characterised by social deficits (e.g., autism spectrum disorder and schizophrenia). Stress and sex steroids exert demonstrable effects on mood and cognition. In patients with depression and anxiety disorders, initial attempts to lower/enhance such hormones have thus been undertaken within conventional therapies in order to improve outcomes. Finally, hunger and satiety hormones may be involved in the vicious circle of dysfunctional eating behaviours and weight loss/gain in anorexia or bulimia nervosa.

Conclusion: Three conclusions can be drawn from this review: First, endocrine research should be considered when patients and clinicians are developing multidimensional illness models together. Second, endocrine markers can complement conventional assessments to provide a more comprehensive account of a patient's current state. Third, endocrine testing may guide treatment choices and inform the development of novel treatments.

\section{Keywords}

anxiety, cognition, depression, hormones, mental disorders, mood, psychological therapy 


\section{Highlights}

- Hormones are intrinsic to the development and maintenance of mental disorders

- Endocrine research should be incorporated into multidimensional illness models

- Endocrine markers can complement conventional diagnostic assessments

- Endocrine testing may guide treatment choices and inform the development of new treatments

Psychoneuroendocrinology is an interdisciplinary research area dedicated to the interaction between the mind, brain, and hormonal system (Wolf \& Saucier, 2013). Stress and lifestyle behaviours (e.g., diet, physical activity) are the most frequently studied psychological factors exerting short- and long-term effects on hormones. Conversely, a number of hormones are known to impact on psychological domains, such as cognition, emotions, and behaviour. Among these are hormones involved in social interaction (e.g., oxytocin), stress hormones (e.g., noradrenaline and cortisol), sex hormones (e.g., testosterone, oestradiol, and progesterone), and hormones involved in hunger and satiety (e.g., ghrelin, leptin, or insulin). Given that mental disorders are characterised by abnormalities in cognition, emotions, and behaviour, clinical psychologists may benefit from learning more about alterations in endocrine systems, how they may contribute to symptoms commonly experienced by patients, and how such knowledge may be put to use in clinical practice.

The aim of the present scientific update was therefore to provide a brief overview of endocrine research relevant to the aetiology, diagnostics, and psychopharmacological and psychotherapeutic treatment of mental disorders, including some of the latest studies in this area. Findings will be presented separately for each of the aforementioned domains and summarised in the final part of the manuscript alongside recommendations for clinical applications.

\section{How Hormones Affect Social Interaction}

The most prominent hormone regulating social interaction is oxytocin (MeyerLindenberg, Domes, Kirsch, \& Heinrichs, 2011). Although oxytocin is available in the periphery (e.g., in reproductive organs), its central origin is in the hypothalamus, and oxytocin receptors are widely expressed in numerous areas of the brain, including the frontal cortex, amygdala, and olfactory nucleus.

Oxytocin has demonstrable effects on social cognition and behaviour, such as enhancing theory of mind, emotional recognition, empathy, social exploration, and attachment 
(see also Ditzen et al., 2009; Heinrichs, Baumgartner, Kirschbaum, \& Ehlert, 2003). As such, it has attracted the interest of researchers studying mental disorders characterised by social deficits, including autism spectrum disorders and schizophrenia. There is now evidence to suggest that oxytocin may be an aetiological factor in autism spectrum disorder, since several polymorphisms within the gene encoding its receptor (OXTR) have been found to be risk-inducing (Kranz et al., 2016; LoParo \& Waldman, 2015). On the other hand, peripheral levels of oxytocin are often found to be unaltered in the same patients as well as in patients with psychotic disorders (Rutigliano et al., 2016). These null-findings need to be interpreted with caution, however, since the bioavailability of oxytocin in blood, urine, or saliva does not necessarily reflect its centrally circulating levels (Valstad et al., 2017), which are of greater importance considering the clinical features of these illnesses. Importantly, central levels are only quantifiable in humans via access to the cerebrospinal fluid by means of lumbar puncture. Thus, although it is unlikely that oxytocin levels will be used as a diagnostic illness marker in the near future, their potential role in the pathophysiology of disorders characterised by social deficits can be incorporated into psychoeducation delivered to patients and their next of kin.

Another line of research has examined the effects of intranasally administered oxytocin in patients with autism spectrum disorder and schizophrenia (Keech, Crowe, \& Hocking, 2018), with findings of small but significant improvements in theory of mind (but not in emotion recognition or empathy). In other words, patients receiving exogenous oxytocin showed an increased ability to attribute mental states to others, thus providing ex juvantibus evidence for an involvement of the oxytocin system in the pathophysiology of these disorders. Importantly, numerous open questions need to be answered before oxytocin can be considered as an adjunct treatment for autism spectrum disorder or schizophrenia, including its precise mechanisms of action in the brain, its long-term efficacy, and potential adverse effects (e.g., increased irritability).

Interestingly, more recent research has explored the role of endogenous oxytocin as a modulator of treatment outcomes. A pilot study was able to demonstrate that the lower depressed patients' pre-treatment oxytocin levels, the lower their degree of change in a self-report measure of depression over the course of psychological therapy (Jobst et al., 2018). This finding aligns well with another study in patients with depressive disorders, which showed that oxytocin levels fluctuated during therapy sessions, and in parallel with subtle changes in the therapeutic alliance (i.e., ruptures; Zilcha-Mano, Porat, Dolev, \& Shamay-Tsoory, 2018). Together, these studies suggest that oxytocin levels may also be a useful prognostic tool as well as a means to monitor treatment progress. 


\section{How Hormones Affect Cognition, Mood, and Sexual Function}

Up to now, the largest share of clinical neuroendocrine research has been dedicated to the stress hormones noradrenaline and cortisol. The catecholamine noradrenaline (NA) is available in the brain and in several other body tissues (Fischer \& Nater, 2015). Its central origin is the locus coeruleus, with $\alpha$ - and $\beta$-adrenergic receptors expressed in numerous other brain areas, such as the cortex, thalamus, hippocampus, amygdala, and hypothalamus. In the periphery, NA, together with adrenaline, is the main end product of the sympathetic nervous system, and its receptors are present in all major organs and cells of the immune system.

Noradrenaline has effects on multiple domains of psychological functioning, including cognition, affect, arousal, and pain perception. It is thus unsurprising that 1) cognitive symptoms (e.g., deficits in working memory), as experienced, for instance, by patients with depressive disorders (Maletic, Eramo, Gwin, Offord, \& Duffy, 2017), 2) anxiety and hyperarousal, representing key clinical features of panic disorder and post-traumatic stress disorder (Bandelow et al., 2017), and 3) bodily symptoms such as fatigue and pain, which feature prominently in somatic symptom disorders (Nater, Fischer, \& Ehlert, 2011), are all paralleled by altered NA functioning. Notably, findings are highly complex; depending on the tissue (i.e., different areas within the brain, blood), both elevated and attenuated concentrations of NA are observed, sometimes within the same patient cohort.

Drugs targeting the NA system (e.g., venlafaxine) constitute effective antidepressants and anxiolytics (e.g., Bandelow et al., 2014; DGPPN et al., 2015), thus adding further evidence to the assumption that the NA system is instrumental in the pathophysiology of depressive and anxiety disorders. Clinicians administering such drugs are advised to explain the role of NA to patients before initiating treatment. By contrast, in somatic symptom disorders and in physical diseases, it is mainly the peripheral actions of NA (e.g., its effects on the musculoskeletal or cardiovascular system) which are critical. Interestingly, in patients undergoing coronary artery bypass graft surgery, it was recently shown that preoperative psychological interventions led to significantly lower (i.e., more adaptive) levels of adrenaline after surgery when compared to standard medical care (Salzmann et al., 2017). This finding highlights the value of catecholamines as markers of therapeutic efficacy. Notably, NA activity can also be determined non-invasively, namely via salivary alpha-amylase, an enzyme involved in the digestion of starch, thus facilitating its use in clinical practice (Nater \& Rohleder, 2009).

The glucocorticoid cortisol, the other major stress hormone, is the end point of the hypothalamic-pituitary-adrenal (HPA) axis (Ehlert, 2011). Although cortisol is synthesised in the adrenal cortex, both central (e.g., the hippocampus) and peripheral tissues (e.g., lymphocytes) are densely populated by mineralocorticoid and glucocorticoid recep- 
tors. Apart from the gluconeogenetic and anti-inflammatory effects of cortisol, one of its main effects lies in influencing cognition.

Akin to the findings on NA, cortisol concentrations have been found to be abnormal in a number of patients presenting with cognitive problems (e.g., difficulty concentrating), as is the case in affective disorders (Belvederi Murri et al., 2016; Stetler \& Miller, 2011). Moreover, abnormal cortisol concentrations have been demonstrated in patients with post-traumatic stress disorder, where they may contribute to re-experiencing of trauma via constant retrieval of the fear memory (Morris, Compas, \& Garber, 2012), and in somatic symptom disorders, where they may contribute to bodily complaints (Tak et al., 2011). Interestingly, while patients with affective disorders are characterised by comparably elevated levels of cortisol (i.e., hypercortisolism), patients with posttraumatic stress disorder or somatic symptom disorders mostly exhibit diminished levels (i.e., $h y$ pocortisolism; Ehlert, Gaab, \& Heinrichs, 2001; Heim, Ehlert, \& Hellhammer, 2000). These differences may be attributable to different genetic predispositions and/or different amounts of stress experienced during the lifespan (Ehlert, 2013). Together, these findings underline the potential of cortisol for improving differential diagnostics.

These findings have been extended by clinical studies, where a similar dichotomy appears to prevail. In depression, the current state of research suggests that the higher a patient's pre-treatment cortisol levels, the lower their chances of responding to psychological therapy (Fischer, Strawbridge, Herane Vives, \& Cleare, 2017). In addition, initial attempts to improve memory performance in these patients have been undertaken using agents which act on glucocorticoid receptors (e.g., mifepristone) or influence cortisol synthesis (e.g., ketoconazole; Soria et al., 2018). In anxiety disorders, the pattern seems to be reversed, insofar as lower cortisol levels predict worse treatment outcomes (Fischer \& Cleare, 2017), although importantly, this only appears to be true for stimulated cortisol as measured during exposure sessions. This has been interpreted as an implication that a certain amount of cortisol is a prerequisite for patients to form an extinction memory, which is one of the key mechanisms underlying successful treatment for fear-related illnesses. More recent research suggests that this knowledge may be utilised to optimise exposure-based psychological therapy for anxiety disorders. For instance, Meuret et al. (2016) were able to demonstrate that early-day exposure sessions (i.e., when endogenous cortisol levels are highest) led to greater clinical improvement in patients with panic disorder and agoraphobia when compared to sessions held later on during the day.

Sex hormones, such as testosterone, oestradiol, and progesterone, are end products of the hypothalamic-pituitary-gonadal (HPG) axis (Melcangi, Giatti, \& Garcia-Segura, 2016). They are mainly produced in the testes in men and in the ovaries in women, and their key function is to orchestrate reproduction and sexual functioning. However, sex steroids also act as neurosteroids (e.g., fostering neurogenesis and differentiation) in various parts of the brain, such as the hippocampus and prefrontal cortex. 
In terms of mental disorders, the bulk of research to date has studied the role of sex steroids in sexual dysfunctions. Testosterone, for instance, is lowered in men with erectile disorder (Isidori et al., 2014), and hormonal (replacement) therapy has been proven to be useful in enhancing erectile function in hypogonadal men (Corona et al., 2017; Elliott et al., 2017) and sexual function in post-menopausal women (Elraiyah et al., 2014). However, long-term follow-up studies are scarce and potential adverse effects of exogenous testosterone (e.g., acne) need to be carefully weighed against the benefits. Similarly, oestrogens and combined oestrogen/progestogen treatments appear to enhance sexual function in some post-menopausal women (Nastri et al., 2013), but again, side effects need to be considered. These findings are important for any clinical psychologist advising patients with sexual dysfunctions in terms of adjunct treatments.

A burgeoning literature also demonstrates the involvement of sex hormones in other mental disorders, which is attributable to the aforementioned central expression of steroid receptors. In schizophrenia, it was shown that oestradiol and selective oestradiol receptor modulators (e.g., raloxifene) can enhance memory and executive functions (Soria et al., 2018). In addition, recent research suggests that sex steroids may exert positive effects on mood. For instance, longer lifetime exposure to endogenous and exogenous oestradiol was found to be linked to fewer depressive symptoms during the menopausal transition (Marsh et al., 2017), whereas greater fluctuations in endogenous oestradiol during the menopausal transition predicted more depressive symptoms in women reporting high amounts of stress (Gordon, Rubinow, Eisenlohr-Moul, Leserman, \& Girdler, 2016). These findings not only contribute to a more profound understanding of the symptoms pertaining to psychotic and mood disorders, but may ultimately be put to use in order to guide (sex-oriented) treatment choices.

\section{How Hormones Affect Hunger and Satiety}

A number of hormones regulating hunger and satiety, such as ghrelin, leptin, or insulin, have been related to different mental disorders (Drobnjak \& Ehlert, 2011). Whereas the orexigenic hormone ghrelin is produced in the stomach, the anorexic hormones leptin and insulin are produced in adipose tissue and in the pancreas, respectively. All three hormones are capable of crossing the blood-brain barrier and thus directly influence energy homoeostasis by acting on the hypothalamus.

Ghrelin, leptin, and insulin have, for the most part, been objects of research into eating disorders. For instance, enhanced baseline ghrelin levels were reported in patients with eating disorders (Prince, Brooks, Stahl, \& Treasure, 2009), likely as a consequence of restrained eating. Importantly, elevated levels of ghrelin may in turn facilitate other dysfunctional behaviours, such as hoarding food in anorexia nervosa or binge eating in bulimia nervosa or binge eating disorder. Furthermore, patients with anorexia nervosa have been found to present with increased insulin sensitivity, whereas patients with bulimia 
nervosa or binge eating disorder exhibit decreased insulin sensitivity (Ilyas et al., 2018). Similar to ghrelin, these findings have been interpreted as being the result of dietary restriction and weight loss/weight gain, respectively, while at the same time further contributing to dysfunctional eating patterns by affecting appetite regulation in the brain (i.e., diminishing/enhancing appetite). This is important knowledge when trying to make sense of the vicious circles that perpetuate eating disorders.

Evidence is now also accumulating that hunger and satiety hormones are abnormal in other mental disorders, mainly those presenting with metabolic symptoms and/or comorbid metabolic diseases (e.g., diabetes mellitus). Findings include elevated levels of leptin and insulin resistance in patients with psychotic disorders (Greenhalgh et al., 2017; Pillinger et al., 2017; Stubbs, Wang, Vancampfort, \& Miller, 2016) and depressive disorders (Kan et al., 2013). Notably, these seem to be independent of BMI and intake of antipsychotic medication (which are known to have several metabolic side effects). This suggests that these hormonal abnormalities are not a mere consequence of lifestyle behaviours associated with suffering from a chronic illness, but may be antecedents of highly debilitating ancillary symptoms pertaining to psychotic and depressive disorders.

Importantly, recent studies support the notion that endogenous ghrelin and leptin may also influence treatment outcomes: Whereas increases in ghrelin predicted non-responses to treatment with lithium-augmented antidepressants in patients with depression (Ricken et al., 2017), leptin was positively linked to increases in BMI (Ricken et al., 2016). In terms of ghrelin, the observed increases in non-responders could be interpreted as being secondary to reduced appetite, a core symptom of severe depression. In terms of leptin, synergistic actions with lithium on the serotonergic system could have resulted in an attenuation of leptin's anorexic effect, but more research is warranted to investigate the intricate interplay between the two systems. These findings are important to consider by clinicians prescribing psychoactive drugs, and will hopefully allow the adjusting of treatments to the needs of the individual patient in the future.

\section{Summary and Integration}

It is evident from this brief overview that hormones are intrinsic to both the development and maintenance of mental disorders, and there are several conceivable ways in which this knowledge may be useful to clinical psychology. First, neuroendocrine research should find its way into clinical practice when clinicians and patients are developing multidimensional illness models together, such as at the beginning of psychological therapy. This is important given that mental disorders are still stigmatised by a large proportion of the general population due to lay concepts about their origins (e.g., depressive disorders being seen as a lack of willpower). Second, endocrine markers may be used to aid the (differential) diagnostics of mental disorders. This is important in light of the fact that not all aspects of mental health are accessible by means of introspection, let alone by in- 
dividuals who suffer from deficits in detecting and reporting signs of psychological distress (e.g., those scoring high on alexithymia). Similarly, hormones may be used to assess treatment outcomes above and beyond self-report symptom measures or clinical rating scales. These ideas align well with the US National Institute of Mental Health (NIMH) Research Domain Criteria (RDoC), which aim to provide more precise characterisations of a patient by integrating biological and psychological research (e.g., Insel et al., 2010). To this end, a matrix combining five psychological domains (social processes, arousal/regulation, negative valence, positive valence, and cognition) with different units of analysis (genes, molecules, cells, neural circuits, physiology, behaviour, self-reported information, and paradigms) has been proposed. This allows for a particular state of mental illness to be described by deficits in different psychological domains, which map on to specific biological substrates (e.g., neuroendocrine abnormalities). Third, the results of neuroendocrine testing may guide treatment choices, that is, they may support clinicians in finding out what is likely to work for whom and why. This resonates well with the central tenet of precision psychiatry, which advocates the tailoring of treatments to the needs of the individual patient by integrating data from multiple levels of information (e.g., biological, personality, and behavioural measures). On a related note, alternative or additional treatments for mental disorders may be developed that are based on a more in-depth account of patients' pathophysiology (e.g., hormonal substitution as an augmentation to psychological therapy).

In the foreseeable future, clinical psychology is likely to benefit from a number of emerging trends in psychoneuroendocrinological research. Elucidating the genetic and epigenetic underpinnings of endocrine functioning will be crucial to fully comprehend its role in mental disorders. As both the distribution and sensitivity of endocrine receptors are governed by genetic variation as well as by the individual's epigenetic make-up (e.g., DNA methylation), this could ultimately enable the identification of patients who run the risk of developing mental illnesses. Similarly, learning more about the cross-talk between different endocrine systems and between endocrine and other bodily systems (e.g., central monoaminergic systems) should allow for a more accurate description of how, precisely, endocrine disturbances contribute to the onset of mental disorders - and provide more precise targets for additional or alternative treatment options. Finally, the advent of novel methodologies to assess hormones in a reliable, non-invasive manner (e.g., fingernail cortisol) holds the promise to translate neuroendocrine knowledge into clinical practice - and hopefully to the benefit of patients and clinical psychologists alike.

Funding: The authors received no financial support for the research, authorship, and/or publication of this article.

Competing Interests: The authors declare no conflicts of interest.

Acknowledgments: The authors have no support to report. 


\section{References}

Bandelow, B., Baldwin, D., Abelli, M., Bolea-Alamanac, B., Bourin, M., Chamberlain, S. R., .. . Riederer, P. (2017). Biological markers for anxiety disorders, OCD and PTSD: A consensus statement. Part II: Neurochemistry, neurophysiology and neurocognition. The World fournal of Biological Psychiatry, 18(3), 162-214. https://doi.org/10.1080/15622975.2016.1190867

Bandelow, B., Wiltink, J., Alpers, G. W., Benecke, C., Deckert, J., Eckhardt-Henn, A., . . Beutel, M. E. (2014). Deutsche S3-Leitlinie Behandlung von Angststörungen. Retrieved from www.awmf.org/leitlinien.html

Belvederi Murri, M., Prestia, D., Mondelli, V., Pariante, C., Patti, S., Olivieri, B., . . Amore, M. (2016). The HPA axis in bipolar disorder: Systematic review and meta-analysis. Psychoneuroendocrinology, 63, 327-342. https://doi.org/10.1016/j.psyneuen.2015.10.014

Corona, G., Rastrelli, G., Morgentaler, A., Sforza, A., Mannucci, E., \& Maggi, M. (2017). Metaanalysis of results of testosterone therapy on sexual function based on international index of erectile function scores. European Urology, 72(6), 1000-1011. https://doi.org/10.1016/j.eururo.2017.03.032

DGPPN, BÄK, KBV, AWMF, AkdÄ, BPtK, ... DGRW (2015). Unipolare Depression (S3-Leitlinie/ Nationale VersorgungsLeitlinie, Version 5, 2nd ed.). Berlin, Germany: ÄZQ.

Ditzen, B., Schaer, M., Gabriel, B., Bodenmann, G., Ehlert, U., \& Heinrichs, M. (2009). Intranasal oxytocin increases positive communication and reduces cortisol levels during couple conflict. Biological Psychiatry, 65(9), 728-731. https://doi.org/10.1016/j.biopsych.2008.10.011

Drobnjak, S., \& Ehlert, U. (2011). Hunger- und Sättigungsregulation. In U. Ehlert \& R. von Känel (Eds.), Psychoendokrinologie und Psychoimmunologie (pp. 151-162). Heidelberg, Germany: Springer.

Ehlert, U. (2011). Das endokrine System. In U. Ehlert \& R. von Känel (Eds.), Psychoendokrinologie und Psychoimmunologie (pp. 3-36). Heidelberg, Germany: Springer.

Ehlert, U. (2013). Enduring psychobiological effects of childhood adversity. Psychoneuroendocrinology, 38(9), 1850-1857. https://doi.org/10.1016/j.psyneuen.2013.06.007

Ehlert, U., Gaab, J., \& Heinrichs, M. (2001). Psychoneuroendocrinological contributions to the etiology of depression, posttraumatic stress disorder, and stress-related bodily disorders: The role of the hypothalamus-pituitary-adrenal axis. Biological Psychology, 57(1-3), 141-152. https://doi.org/10.1016/S0301-0511(01)00092-8

Elliott, J., Kelly, S. E., Millar, A. C., Peterson, J., Chen, L., Johnston, A., . . Wells, G. A. (2017). Testosterone therapy in hypogonadal men: A systematic review and network meta-analysis. BMF Open, 7(11), Article e015284.

Elraiyah, T., Sonbol, M. B., Wang, Z., Khairalseed, T., Asi, N., Undavalli, C., . . Murad, M. H. (2014). Clinical review: The benefits and harms of systemic testosterone therapy in postmenopausal women with normal adrenal function: A systematic review and meta-analysis. The fournal of Clinical Endocrinology \& Metabolism, 99(10), 3543-3550. https://doi.org/10.1210/jc.2014-2262 
Fischer, S., \& Cleare, A. J. (2017). Cortisol as a predictor of psychological therapy response in anxiety disorders: Systematic review and meta-analysis. fournal of Anxiety Disorders, 47, 60-68. https://doi.org/10.1016/j.janxdis.2017.02.007

Fischer, S., \& Nater, U. M. (2015). Autonomes Nervensystem. In W. Rief \& P. Henningsen (Eds.), Psychosomatik und Verhaltensmedizin (pp. 193-201). Stuttgart, Germany: Schattauer.

Fischer, S., Strawbridge, R., Herane Vives, A., \& Cleare, A. J. (2017). Cortisol as a predictor of psychological therapy response in depressive disorders: Systematic review and meta-analysis. The British fournal of Psychiatry, 210(2), 105-109. https://doi.org/10.1192/bjp.bp.115.180653

Gordon, J. L., Rubinow, D. R., Eisenlohr-Moul, T. A., Leserman, J., \& Girdler, S. S. (2016). Estradiol variability, stressful life events, and the emergence of depressive symptomatology during the menopausal transition. Menopause, 23(3), 257-266.

https://doi.org/10.1097/GME.0000000000000528

Greenhalgh, A. M., Gonzalez-Blanco, L., Garcia-Rizo, C., Fernandez-Egea, E., Miller, B., Arroyo, M. B., \& Kirkpatrick, B. (2017). Meta-analysis of glucose tolerance, insulin, and insulin resistance in antipsychotic-naive patients with nonaffective psychosis. Schizophrenia Research, 179, 57-63. https://doi.org/10.1016/j.schres.2016.09.026

Heim, C., Ehlert, U., \& Hellhammer, D. H. (2000). The potential role of hypocortisolism in the pathophysiology of stress-related bodily disorders. Psychoneuroendocrinology, 25(1), 1-35. https://doi.org/10.1016/S0306-4530(99)00035-9

Heinrichs, M., Baumgartner, T., Kirschbaum, C., \& Ehlert, U. (2003). Social support and oxytocin interact to suppress cortisol and subjective responses to psychosocial stress. Biological Psychiatry, 54(12), 1389-1398. https://doi.org/10.1016/S0006-3223(03)00465-7

Ilyas, A., Hubel, C., Stahl, D., Stadler, M., Ismail, K., Breen, G., . . Kan, C. (2018). The metabolic underpinning of eating disorders: A systematic review and meta-analysis of insulin sensitivity. Molecular and Cellular Endocrinology. Advance online publication.

https://doi.org/10.1016/j.mce.2018.10.005

Insel, T., Cuthbert, B., Garvey, M., Heinssen, R., Pine, D. S., Quinn, K., . . Wang, P. (2010). Research domain criteria $(\mathrm{RDoC})$ : Toward a new classification framework for research on mental disorders. The American fournal of Psychiatry, 167(7), 748-751.

https://doi.org/10.1176/appi.ajp.2010.09091379

Isidori, A. M., Buvat, J., Corona, G., Goldstein, I., Jannini, E. A., Lenzi, A., . . Maggi, M. (2014). A critical analysis of the role of testosterone in erectile function: From pathophysiology to treatment-a systematic review. European Urology, 65(1), 99-112.

https://doi.org/10.1016/j.eururo.2013.08.048

Jobst, A., Sabass, L., Hall, D., Brucklmeier, B., Buchheim, A., Hall, J., . . Padberg, F. (2018). Oxytocin plasma levels predict the outcome of psychotherapy: A pilot study in chronic depression. Journal of Affective Disorders, 227, 206-213. https://doi.org/10.1016/j.jad.2017.10.037

Kan, C., Silva, N., Golden, S. H., Rajala, U., Timonen, M., Stahl, D., \& Ismail, K. (2013). A systematic review and meta-analysis of the association between depression and insulin resistance. Diabetes Care, 36(2), 480-489. https://doi.org/10.2337/dc12-1442 
Keech, B., Crowe, S., \& Hocking, D. R. (2018). Intranasal oxytocin, social cognition and neurodevelopmental disorders: A meta-analysis. Psychoneuroendocrinology, 87, 9-19. https://doi.org/10.1016/j.psyneuen.2017.09.022

Kranz, T. M., Kopp, M., Waltes, R., Sachse, M., Duketis, E., Jarczok, T. A., . . Chiocchetti, A. G. (2016). Meta-analysis and association of two common polymorphisms of the human oxytocin receptor gene in autism spectrum disorder. Autism Research, 9(10), 1036-1045. https://doi.org/10.1002/aur.1597

LoParo, D., \& Waldman, I. D. (2015). The oxytocin receptor gene (OXTR) is associated with autism spectrum disorder: A meta-analysis. Molecular Psychiatry, 20(5), 640-646.

https://doi.org/10.1038/mp.2014.77

Maletic, V., Eramo, A., Gwin, K., Offord, S. J., \& Duffy, R. A. (2017). The role of norepinephrine and its alpha-adrenergic receptors in the pathophysiology and treatment of major depressive disorder and schizophrenia: A systematic review. Frontiers in Psychiatry, 8, Article 42. https://doi.org/10.3389/fpsyt.2017.00042

Marsh, W. K., Bromberger, J. T., Crawford, S. L., Leung, K., Kravitz, H. M., Randolph, J. F., . . Soares, C. N. (2017). Lifelong estradiol exposure and risk of depressive symptoms during the transition to menopause and postmenopause. Menopause, 24(12), 1351-1359. https://doi.org/10.1097/GME.0000000000000929

Melcangi, R. C., Giatti, S., \& Garcia-Segura, L. M. (2016). Levels and actions of neuroactive steroids in the nervous system under physiological and pathological conditions: Sex-specific features. Neuroscience \& Biobehavioral Reviews, 67, 25-40. https://doi.org/10.1016/j.neubiorev.2015.09.023

Meuret, A. E., Rosenfield, D., Bhaskara, L., Auchus, R., Liberzon, I., Ritz, T., \& Abelson, J. L. (2016). Timing matters: Endogenous cortisol mediates benefits from early-day psychotherapy. Psychoneuroendocrinology, 74, 197-202. https://doi.org/10.1016/j.psyneuen.2016.09.008

Meyer-Lindenberg, A., Domes, G., Kirsch, P., \& Heinrichs, M. (2011). Oxytocin and vasopressin in the human brain: Social neuropeptides for translational medicine. Nature Reviews Neuroscience, 12(9), 524-538. https://doi.org/10.1038/nrn3044

Morris, M. C., Compas, B. E., \& Garber, J. (2012). Relations among posttraumatic stress disorder, comorbid major depression, and HPA function: A systematic review and meta-analysis. Clinical Psychology Review, 32(4), 301-315. https://doi.org/10.1016/j.cpr.2012.02.002

Nastri, C. O., Lara, L. A., Ferriani, R. A., Rosa, E. S. A. C., Figueiredo, J. B., \& Martins, W. P. (2013). Hormone therapy for sexual function in perimenopausal and postmenopausal women. Cochrane Database of Systematic Reviews, 6, Article CD009672.

Nater, U. M., Fischer, S., \& Ehlert, U. (2011). Stress as a pathophysiological factor in functional somatic syndromes. Current Psychiatry Reviews, 7(2), 152-169. https://doi.org/10.2174/157340011796391184

Nater, U. M., \& Rohleder, N. (2009). Salivary alpha-amylase as a non-invasive biomarker for the sympathetic nervous system: Current state of research. Psychoneuroendocrinology, 34(4), 486-496. https://doi.org/10.1016/j.psyneuen.2009.01.014 
Pillinger, T., Beck, K., Gobjila, C., Donocik, J. G., Jauhar, S., \& Howes, O. D. (2017). Impaired glucose homeostasis in first-episode schizophrenia: A systematic review and meta-analysis. JAMA Psychiatry, 74(3), 261-269. https://doi.org/10.1001/jamapsychiatry.2016.3803

Prince, A. C., Brooks, S. J., Stahl, D., \& Treasure, J. (2009). Systematic review and meta-analysis of the baseline concentrations and physiologic responses of gut hormones to food in eating disorders. The American fournal of Clinical Nutrition, 89(3), 755-765.

https://doi.org/10.3945/ajcn.2008.27056

Ricken, R., Bopp, S., Schlattmann, P., Himmerich, H., Bschor, T., Richter, C., . . Adli, M. (2017). Ghrelin serum concentrations are associated with treatment response during lithium augmentation of antidepressants. International fournal of Neuropsychopharmacology, 20(9), 692-697. https://doi.org/10.1093/ijnp/pyw082

Ricken, R., Bopp, S., Schlattmann, P., Himmerich, H., Bschor, T., Richter, C., . . Adli, M. (2016). Leptin serum concentrations are associated with weight gain during lithium augmentation. Psychoneuroendocrinology, 71, 31-35. https://doi.org/10.1016/j.psyneuen.2016.04.013

Rutigliano, G., Rocchetti, M., Paloyelis, Y., Gilleen, J., Sardella, A., Cappucciati, M., . . Fusar-Poli, P. (2016). Peripheral oxytocin and vasopressin: Biomarkers of psychiatric disorders? A comprehensive systematic review and preliminary meta-analysis. Psychiatry Research, 241, 207-220. https://doi.org/10.1016/j.psychres.2016.04.117

Salzmann, S., Euteneuer, F., Laferton, J. A. C., Auer, C. J., Shedden-Mora, M. C., Schedlowski, M., . . . Rief, W. (2017). Effects of preoperative psychological interventions on catecholamine and cortisol levels after surgery in coronary artery bypass graft patients: the randomized controlled PSY-HEART trial. Psychosomatic Medicine, 79(7), 806-814.

https://doi.org/10.1097/PSY.0000000000000483

Soria, V., Gonzalez-Rodriguez, A., Huerta-Ramos, E., Usall, J., Cobo, J., Bioque, M., . . Labad, J. (2018). Targeting hypothalamic-pituitary-adrenal axis hormones and sex steroids for improving cognition in major mood disorders and schizophrenia: A systematic review and narrative synthesis. Psychoneuroendocrinology, 93, 8-19. https://doi.org/10.1016/j.psyneuen.2018.04.012

Stetler, C., \& Miller, G. E. (2011). Depression and hypothalamic-pituitary-adrenal activation: A quantitative summary of four decades of research. Psychosomatic Medicine, 73(2), 114-126. https://doi.org/10.1097/PSY.0b013e31820ad12b

Stubbs, B., Wang, A. K., Vancampfort, D., \& Miller, B. J. (2016). Are leptin levels increased among people with schizophrenia versus controls? A systematic review and comparative metaanalysis. Psychoneuroendocrinology, 63, 144-154. https://doi.org/10.1016/j.psyneuen.2015.09.026

Tak, L. M., Cleare, A. J., Ormel, J., Manoharan, A., Kok, I. C., Wessely, S., \& Rosmalen, J. G. M. (2011). Meta-analysis and meta-regression of hypothalamic-pituitary-adrenal axis activity in functional somatic disorders. Biological Psychiatry, 87(2), 183-194. https://doi.org/10.1016/j.biopsycho.2011.02.002

Valstad, M., Alvares, G. A., Egknud, M., Matziorinis, A. M., Andreassen, O. A., Westlye, L. T., \& Quintana, D. S. (2017). The correlation between central and peripheral oxytocin concentrations: 
A systematic review and meta-analysis. Neuroscience \& Biobehavioral Reviews, 78, 117-124. https://doi.org/10.1016/j.neubiorev.2017.04.017

Wolf, J. M., \& Saucier, E. (2013). Psychoneuroendocrinology. In M. D. Gellman \& J. R. Turner (Eds.), Encyclopedia of Behavioral Medicine. New York, NY, USA: Springer. https://doi.org/10.1007/978-1-4419-1005-9

Zilcha-Mano, S., Porat, Y., Dolev, T., \& Shamay-Tsoory, S. (2018). Oxytocin as a neurobiological marker of ruptures in the working alliance. Psychotherapy and Psychosomatics, 87(2), 126-127. https://doi.org/10.1159/000487190

\section{EACLIPT}

Clinical Psychology in Europe (CPE) is the official journal of the European Association of Clinical Psychology and Psychological Treatment (EACLIPT).

\section{(P) leibniz-psychology.org}

PsychOpen GOLD is a publishing service by Leibniz Institute for Psychology Information (ZPID), Germany. 